\title{
Beam optimization in a 25 TW femtosecond laser system for high harmonic generation
}

\author{
Jialin $\mathrm{Li}^{1} \odot$, Yang Wang ${ }^{1}$, Tianyi Guo ${ }^{1}$, Jonathon White ${ }^{1}$, \\ Matthew Weidman ${ }^{2}$, Yi Wu ${ }^{1}$, Kai Hu${ }^{1}$, Marieke F Jager ${ }^{3}$, Christopher $\mathbf{J}$ \\ Kaplan $^{3}$, Romain Geneaux ${ }^{3}$, Daniel M Neumark ${ }^{3}$, Stephen R Leone ${ }^{3,4}$, \\ Graham G Brown ${ }^{5}$, Paul Corkum ${ }^{5}$ and Zenghu Chang ${ }^{1,6}$ \\ ${ }^{1}$ Institute for the Frontier of Attosecond Science and Technology, CREOL and Department of Physics, \\ University of Central Florida, Orlando, FL 32816, United States of America \\ 2 Max-Planck-Institut für Quantenoptik, Hans-Kopfermann-Str. 1, D-85748 Garching, Germany \\ ${ }^{3}$ Departments of Chemistry and Physics, University of California, Berkeley, CA 94720, United States of \\ America \\ ${ }^{4}$ Chemical Sciences Division, Lawrence Berkeley National Laboratory, Berkeley, CA 94720, United \\ States of America \\ 5 Joint Attosecond Laboratory, National Research Council and University of Ottawa, Ottawa, ON K1A \\ 0R6, Canada \\ E-mail: Zenghu.Chang@ucf.edu
}

Received 31 December 2019, revised 21 April 2020

Accepted for publication 29 April 2020

Published 18 June 2020

\begin{abstract}
It has been demonstrated in the past that high fluxes of extreme ultraviolet (XUV) light could be obtained by driving high harmonic generation (HHG) with high energy lasers. However, the peak intensity at the focal point of a femtosecond laser with more than $100 \mathrm{~mJ}$ can be too high for phase-matched HHG in gases. We propose a method to optimize the spatial profile at off-focus locations of the high energy driving laser to avoid fully ionizing the target atoms. The beam profile before or after the focal point depends on the wavefront quality of the laser beam and the near field intensity distribution. The beam spot diameter reaches three times that at the focus by adding customized wavefront terms. An XUV pulse energy of $5.6 \mu \mathrm{J}$ was obtained from HHG when a larger off-focus spot of a $500 \mathrm{~mJ}$ Ti: sapphire laser beam was applied in an argon gas cell.
\end{abstract}

Keywords: wavefront correction, high harmonic generation, XUV

(Some figures may appear in colour only in the online journal)

\section{Introduction}

Over the past decades, there has been tremendous progress in attosecond based XUV radiation and its applications, both experimentally [1-5] and theoretically [6, 7]. Schemes to obtain a high flux XUV source have attracted significant attention [8-11]. It opens a way to investigate time-resolved spectroscopy as well as nonlinear phenomena with attosecond resolution. HHG in gas medium is a routine approach to obtain XUV sources at the laboratory level [12]. Having a wellcontrolled driving laser beam helps to promote conversion efficiency from driving laser to XUV.

${ }^{6}$ The author to whom any correspondence should be addressed.
Typically, the temporal duration can be retrieved using a frequency-resolved optical gating (FROG) setup. Experiments have shown the ability to optimize the pulse shape by genetic algorithm [13, 14]. Neural network through deep learning method is also developed, and its application is extended in attosecond pulse characterizations recently [15]. The acousto optic programmable dispersive filter (AOPDF, or DAZZLER) is promising to compensate the phase errors with high order dispersion contributions. A clean pulse shape is thus expected. Optical aberrations are introduced during beam propagation. Efforts have been made to compensate the aberrations and attain an optimized focus of the driving laser [16]. An adaptive 
optics system is employed as an effective scheme for correcting the wavefront aberrations [17, 18]. A diffraction-limited focal spot can be achieved and a laser intensity as high as $10^{20}$ $\mathrm{W} \mathrm{cm}{ }^{-2}$ was obtained [19, 20].

Loose focusing geometry in the high energy laser systems for HHG was proposed and demonstrated for $\sim 100 \mathrm{~mJ}$ level femtosecond driving lasers to obtain a large focal spot size and a long interaction length $[10,21]$. Optimum geometry is achieved with photon numbers $N_{q}$ being proportional to the function that: $N_{q} \sim S_{\text {spot }}\left(P L_{\text {med }}\right)^{2}$ [10], where $S_{\text {spot }}$ is the driving beam area at the generation location, $P$ is the gas pressure at the interaction region, and $L_{\text {med }}$ is the length of gas medium. $S_{\text {spot }}$ and $L_{\text {med }}$ scale up to a few $\mathrm{mm}^{2}$ in area and centimeters in length, respectively. The beam needs to magnify to 50-75 mm in size during propagation before focusing in a high-energy femtosecond system (joule level), to avoid optical damage. The laser beam diameter at the HHG target must be sufficiently large to avoid over-ionizing the target atoms. It then requires hundred-meter focal lengths to achieve the desired focal geometry, which is not practically obtainable on the laboratory scale. A straightforward, but rarely realized routine is to consider the larger beam spot at an off-focus position. The larger spot is preferred to obtain a large generation volume, as well as to maintain a preferable laser peak intensity. However, the beam profile before or after the laser focus typically has structures and distortions, which is not desirable for HHG.

In this paper, we will introduce a scenario to optimize the pulse shape, and more importantly, present a solution to obtain the desired focal geometry in a 25 TW Ti: sapphire laser system $(10 \mathrm{~Hz}$ repetition rate with $500 \mathrm{~mJ}$ pulse energy) using a customized correction of the driving laser wavefront. A tenfold enlargement of the beam spot area was obtained in the HHG gas medium. The larger beam spot was employed for XUV generation, which resulted in an XUV pulse energy of $5.6 \mu \mathrm{J}$.

\section{Experimental setup}

The phase compensation and wavefront control are performed on a near-infrared (NIR) driving laser, which is a $10 \mathrm{~Hz}$ laser system with $790 \mathrm{~nm}$ central wavelength and $500 \mathrm{~mJ}$ pulse energy [16]. Generally, it originates from a multi-pass Ti: sapphire chirped pulse amplification (CPA) system, spectral broadening in a hollow-core fiber, and stretched by an Öffnertype stretcher to $300 \mathrm{ps}$. It is then followed by another two stages of amplification, which are pumped by a Nimma-900 (Beamtech) and two Quanta-Ray PRO-350 (Spectra-Physics) lasers, respectively. Two $200 \mathrm{~mJ}, 1.2 \mathrm{~mm}$, single pass pumps and two $3.5 \mathrm{~J}, 15 \mathrm{~mm}$, single pass pumps were applied in the first stage crystal and the second stage crystal, respectively. The pulse is compressed to within 20 fs using a grating pair and a Dazzler (Fastlite) device. The output beam size is magnified from $25 \mathrm{~mm}$ to $70 \mathrm{~mm}$ through a telescope, and loosely focused 25 meters away, where the gas tube for HHG was placed.

During amplification, high order spectral phase distortions emerge and thermal effects appear, especially from the second

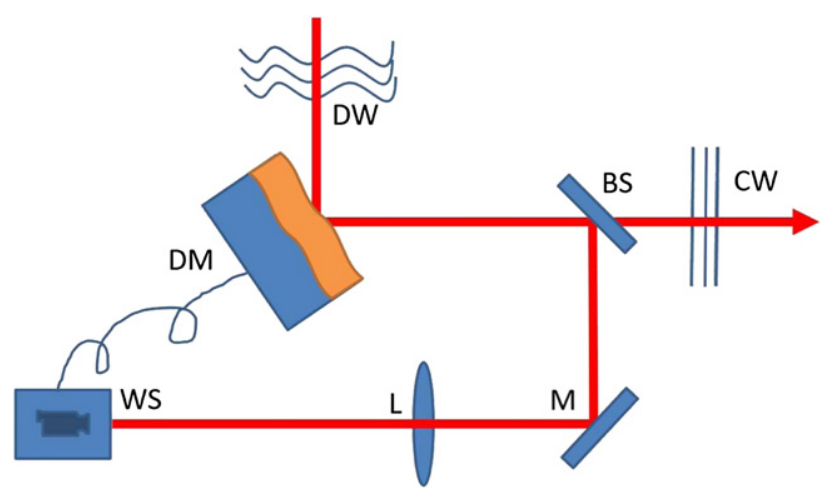

Figure 1. Wavefront correction of the high energy beam in an adaptive optics system. DW: distorted wavefront, CW: corrected wavefront, DM: deformable mirror, WS: wavefront sensor, BS: beam splitter, M: mirror, L: lens, see text for details.

stage. The beam is compressed, sampled, and then measured by a home-built frequency doubling FROG setup. The dispersion terms are compensated up to the fourth order when the genetic algorithm is performed. The focusability relies on the wavefront quality.

Figure 1 shows a schematic diagram of the wavefront measurement and correction setup. A deformable mirror (ILAO star serial, imagine Optic) is set before the grating compressor pair, and a wavefront sensor (HASO3-first, Imagine Optic) is placed subsequently to image the deformable mirror plane position. Voltage settings are applied on 52 mechanical actuators in the deformable mirror with $75 \mathrm{~mm}$ pupil diameter, which modify the reflective surface on a nanometric scale. The deformable mirror acts via its shaped surface to modify the wavefront accordingly. The incidence angle of 45-degree high reflective surface ( $>99 \%$ for S polarization in 700-900 nm) is optimized with a low group velocity dispersion (within $100 \mathrm{fs}^{2}$ in 700-900 $\mathrm{nm}$ regime) and high damage threshold $\left(>5 \mathrm{~J} \mathrm{~cm}^{-2}\right.$ at $10 \mathrm{~Hz}$ repletion rate, $1 \mathrm{~ns}$ pulse width at $800 \mathrm{~nm})$. The wavefront sensor with $4.8 \times 3.6 \mathrm{~mm}$ dimension $(656 \times 494$ pixels $)$ consists of a matrix of microlenses $(40 \times 32)$. It measures the absolute wavefront using Shack-Hartmann technology [17] after precise calibrations through a perfect reference wavefront from a single mode optical fiber laser. A $100 \mathrm{~mm}$ diameter plano-convex lens is utilized $(f=500 \mathrm{~mm})$ with a demagnification ratio of 20 to adapt the relay image from the deformable mirror plane into the wavefront sensor. An electronic control unit was installed to load the wavefront profile from the sensor for monitoring and feedback to the deformable mirror to adjust the voltage accordingly. The sensor was synchronized to the laser pulses, allowing the deformable mirror stabilization $(0.3 \mathrm{~s})$ in finite closed loops. The full power beam was measured by applying attenuation wedges and neutral density filters. The wedges have a high surface quality with flatness within $\lambda / 10$, while the surface of the neutral density filters is in the $\lambda$ range. The wavefront error was manually compensated, as shown in the following section. A charge-coupled device (CCD) was installed during the correction procedure, to monitor the beam spot around the focal position, acting as a replica of the HHG gas tube location. 

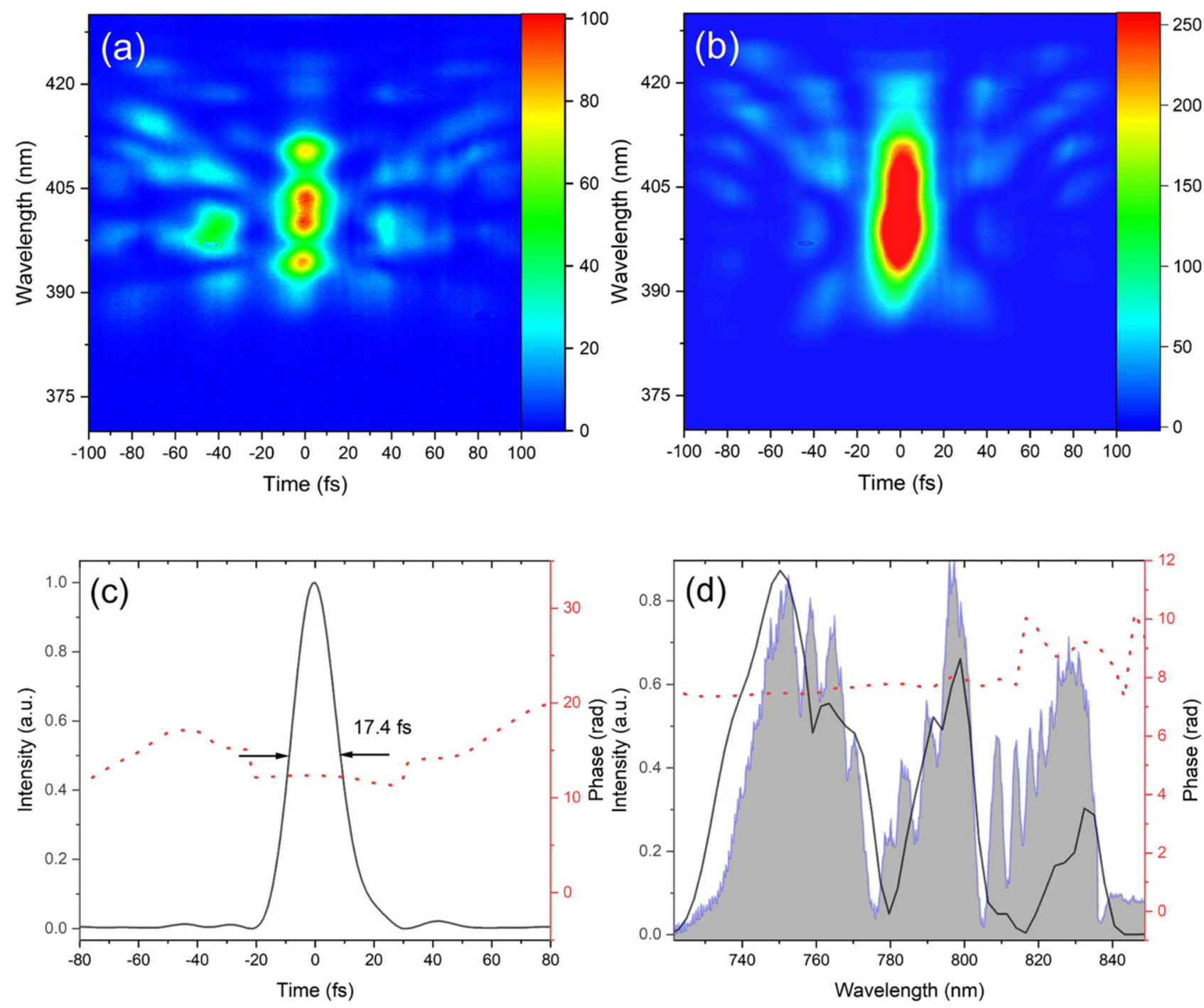

Figure 2. Characterization of the 17.4 fs laser pulse by a second-harmonic generation FROG. (a) The measured FROG trace after genetic algorithm compensation; (b) the updated FROG trace after the spectral phase retrieval and compensation by the DAZZLER; (c) the retrieved pulse shape (solid line) and the temporal phase (red dashed line); (d) the retrieved spectrum (solid black line) and the spectral phase (red dashed line) together with the measured spectrum (filled gray curve).

\section{Results and discussions}

\subsection{Temporal optimization}

A femtosecond pulse with short and clean temporal features is essential where high peak intensity is required in HHG. It is also preferred to gate the generated attosecond pulse train to isolated attosecond pulses. In this work, it was achieved by a genetic algorithm to obtain a uniform FROG trace to get the phase retrieved, and compensated using a DAZZLER.

The genetic algorithm was performed through generations according to a combined factor. The factor includes the time zero range proportion in a random trace, and uniformity through the spectrum. The uniformity is introduced to avoid the local maximization of the FROG signal in a certain spectral range. The FROG trace was preliminarily compressed, especially for the lower dispersion orders, which provides a panoramic signal-level for further analysis.
A typical FROG trace after the genetic algorithm is shown in figure 2(a). The phase was retrieved and the preserved spectral phase curve was sent back to the DAZZLER. Dispersion polynomial orders up to the fourth-order dispersion are included. The characterization of pulse duration and spectral phase information was then performed again after the phase compensation. It may repeat up to three times to achieve an optimal phase correction. An updated FROG trace (figure 2(b)) with 17.4 fs pulse duration was obtained after phase compensation. Both the temporal (figure 2(c)) phase and spectral phase (figure 2(d)) are illustrated. The results were obtained after fifteen iterations of the genetic algorithm with restricted ranges, and two times spectral phase compensation using the DAZZLER. Compensating the high order phase errors helped to reduce the pulse duration, and more importantly to eliminate the pre and post pulses. 


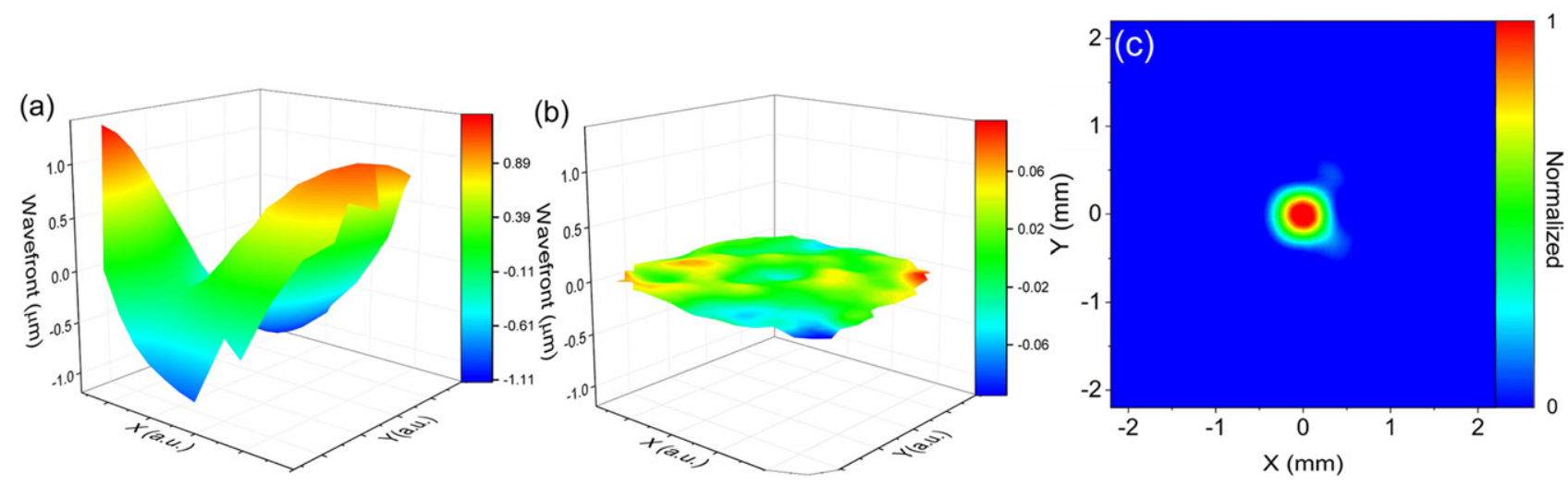

Figure 3. (a) A typical wavefront profile of the driving laser before correction. The defocus term is removed. $635 \mathrm{~nm}$ RMS deviation is noted; (b) the wavefront is corrected to RMS of $30 \mathrm{~nm}(\lambda / 25)$; (c) focal spot is measured after the wavefront loop correction, and the tiny compensated terms are added. A near diffraction-limited spot is obtained.

\subsection{Wavefront correction}

The wavefront aberrations can be described in terms of Zernike polynomials $[22,23]$.

$$
W(r, \varphi)=\sum_{n} \sum_{m=-n}^{n} c_{n m} Z_{n}^{m}(r, \varphi)
$$

where $c_{n m}$ is the Zernike coefficient and $Z_{n}^{m}(r, \varphi)$ is the Zernike polynomial for the $n$th radial and $m$ th azimuthal order. The orders in the $Z_{n}^{m}$ terms represent the orthogonal distributions in the wavefront aberration. For instance, the dominant aberrations are defocus $\left(Z_{2}^{0}\right)$, astigmatism $\left(Z_{2}^{2}\right.$ for 0 degree and $Z_{2}^{-2}$ for 45 degree ), coma ( $Z_{3}^{1}$ for $x$ coma, and $Z_{3}^{-1}$ for $y$ coma) and spherical aberration $\left(Z_{4}^{0}\right)$. The wavefront of the driving laser was measured and corrected using the deformable mirror and wavefront sensor.

A typical wavefront profile before correction is shown in figure 3(a) where the root mean square (RMS) of the Zernike coefficients is $635 \mathrm{~nm}$. The wavefront aberrations were corrected to an acceptable level in the first finite iterations, and became optimal and stable within tens of iterations. With the wavefront correction and loop control, the optimal wavefront aberration (see figure $3(\mathrm{~b})$ ) reduces to RMS of $30 \mathrm{~nm}(\sim \lambda / 25)$. The correction limit of the wavefront originates from the surrounding air flow and thermal drift in the laser amplifiers. The focused beam intensity of a real beam, compared to that of an undisturbed flat wavefront, is given by Strehl ratio. The Strehl ratio improved from 0.56 in the original beam to 0.95 with wavefront correction. This indicates a successful wavefront aberration correction in the laser system.

The focus was measured using a CCD camera $(1200 \times$ 1600 , with $5.5 \mu \mathrm{m}$ pixel size), which is placed 25 meters away from the deformable mirror. Beam profile and energy concentration are poor for the raw focal image without any wavefront correction. With the wavefront loop correction being applied, we noticed that the beam profile was improved but still had some image distortions. This originates from extra optical aberrations introduced during wavefront correction and beam propagation. Astigmatism and coma, etc. inevitably appear when the laser passes through a grating compressor, large diameter mirrors, lenses and optics without high quality surfaces. An optimal focus can be obtained by applying manual compensating terms in astigmatism and coma, which is shown in figure 3(c). A near diffraction-limited beam spot as large as $780 \times 780 \mu \mathrm{m}\left(1 / e^{2}\right.$ diameter $)$ was obtained, where the laser peak intensity reached $10^{16} \mathrm{~W} \mathrm{~cm}^{-2}$. A common gas medium used for high flux HHG is argon gas, which has a relatively higher conversion efficiency compared to lighter rare gases. The ionization threshold for argon is $2.5 \times 10^{14} \mathrm{~W} \mathrm{~cm}^{-2}$ as predicted by the Ammosov-Delone-Krainov model [24]. The phase matching is not favorable to achieve with a high ionization percentage. Additionally, enlargement in the generation volume for HHG is a straightforward method to gain more XUV photons as indicated in the introduction section. It thus requires a larger beam spot at the HHG location: i) to lower the ionization of the gas target with adaptable laser intensity for phase matching, and ii) to increase XUV photon generation volume.

\subsection{Off-focus beam spot}

To achieve the favorable phase matching in HHG with the appropriate laser intensity, a large focusing beam spot is required in the high-energy laser system. The beam spot at the focal position is determined by the $f$-number, for the specific wavelength. One possibility is to reduce the aperture size with an iris, which has a limited spot change and the beam energy is drastically reduced. Using the beam spot at an offfocus position is another option, while there are inevitably imaging distortions at off-focus positions starting with a flat top beam propagating through optical components. A method to optimize the off-focus spot is desirable. It is known that the wavefront correction can be used to control the laser energy concentration and optimize the focus $[16,25]$. We demonstrate the ability to optimize the off-focus beam spot by adding a certain amount of spherical aberrations. It starts with an optimized beam spot at focus. The defocus term $Z_{2}^{0}$ was then added to shift the focal length up to 3 meters. The HHG location was thus revised to either before or after focus. The off-focus spot was optimized by applying spherical aberrations (up to 

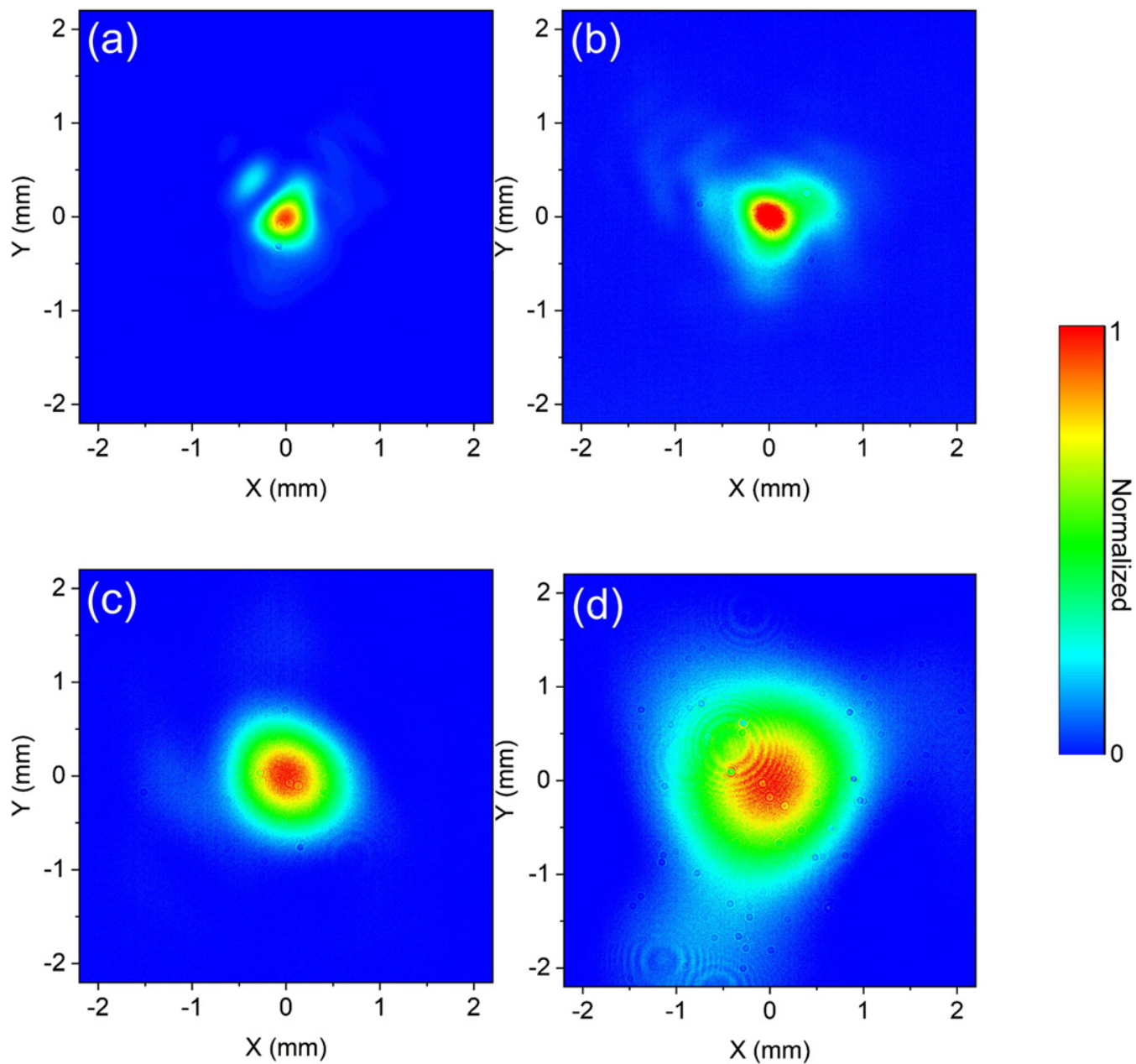

Figure 4. The off-focus beam spots with $406.4 \mathrm{~mm}$ before (a) and after (b) focus. (a): spot diameter $1.1 \times 1.0 \mathrm{~mm}$, and (b): spot diameter $0.95 \times 1.1 \mathrm{~mm}$. They are measured after the focal spot optimization through the wavefront loop correction, without the customized wavefront terms added. (c) and (d) are measured with the defocus and spherical aberrations added. (c): 4.06 meters before the focus with beam diameter $1.6 \times 1.6 \mathrm{~mm}$; (d): 3 meters after the focus and beam diameter is $2.7 \times 3.3 \mathrm{~mm}$. More spherical aberrations are added in (d). See text for details.

$0.3 \mu \mathrm{m}$ ), and tiny astigmatism and coma adjustments for a further improvement. It is observed that the off-focus beam spots were improved remarkably.

Figures 4(a) and (b) show the off-focus beam profiles without adding customized wavefront, while a new series of images at off-focus positions with customized correction are checked, with typical examples shown in figure 4(c) and (d). There are obvious beam distortions with off-focus distance for $406.4 \mathrm{~mm}$ in figure 4(a) and (b). With the extra spherical aberrations applied, images were optimized for positions both before and after focus in $254 \mathrm{~mm}$ steps, distances up to 4.06 meters. The spot size $(1.6 \times 1.6 \mathrm{~mm}$, in figure $4(\mathrm{c}))$ goes up by twice that of the original focal spot. It fits the volume needed for argon gas where the current hundreds of milli-joule laser is applied. With more defocus and appropriate spherical aberrations values, the spot after focus can even reach to $2.7 \times 3.3 \mathrm{~mm}$ (shown in figure 4(d)), which is adaptable to our joule level laser system. The spots exhibit a smooth profile and high energy concertation. It indicates the feasibility to optimize the spot both before focus (up to 4.06 meters) and after focus (up to 3 meters) with good beam profiles. The Rayleigh range increases accordingly to extend the focal depth and fit the gas tube in a ten-centimeter range. The results indicate the HHG generation volume increases by one order of magnitude, and the ability to adapt joule level driving laser.

The temporal profile of the laser pulse is affected by the spherical aberrations added in the wavefront $[25,26]$. Delay time $\tau_{s}$ for the laser pulse passing through certain plane is given by $\tau_{s}=\frac{3 A}{\omega_{0}} r^{4}$, where $\omega_{0}$ is the central frequency, $r$ is the radial coordinate and $A$ is the dimensionless quantity of spherical aberration, which can be given with Zernike coefficient that: $A=6 \sqrt{5} \frac{\omega_{0}}{c} Z_{4}^{0}$, where $c$ is the light speed. To estimate the temporal broadening introduced by spherical aberrations, the statistical average of the delay time is calculated $\tau=\frac{1}{\pi} \int_{0}^{1} \tau_{s} 2 \pi r d r=\frac{A}{\omega_{0}}$. It introduces a temporal broadening of up to $13.4 \mathrm{fs}$ with the spherical aberrations up to $0.3 \mu \mathrm{m}$. The temporal profile was checked again with the FROG setup, providing typically within 20 fs pulse width for the driving laser. It is an acceptable pulse width to generate attosecond pulse trains, and subsequently to obtain isolated attosecond pulse using the generalized double optical gating [27]. The results indicate that optimization of the off-focus beam spot 
was achieved in the high energy laser system, by adding extra spherical aberrations and along with an acceptable temporal broadening.

\subsection{XUV generation}

The development of a high flux attosecond XUV source has been a long-lasting topic. As the high energy driving laser was well controlled with a clean short pulse duration and a large beam spot at off-focus position, the XUV source was obtained by applying the driving laser interacting to the pulsed argon gas. The XUV photons emitted need to be in phase for different locations in the propagation direction, to allow the photons to build up a high flux source. There is an inevitable difference between the phase velocities of the fundamental and high harmonic fields. For a specific harmonic order $q$, the wave-vector mismatch is $\Delta k=\mathrm{q} k_{\mathrm{w}}-k_{q w}$. The phase mismatch $\Delta k$ consists of contributions from plasma and neutral atoms. Phase matching implementation requires a maintenance of various factors. Coherence length $L_{\mathrm{co}}=\pi /|\Delta k|$ is maximized for XUV generation to reach the wave-vector mismatch tending to zero. Two more conceptual lengths are considered besides the coherence length: $L_{\text {abs }}$ responds to $1 /(\alpha \rho)$, where $\alpha$ is absorption coefficient for the specific $q$ th order harmonic, $\rho$ is atomic density; and $L_{\text {med }}$ is the length of gas medium. With the fulfillment of $L_{\mathrm{co}}>5 L_{\mathrm{abs}}$ and longer gas medium $\left(L_{\text {med }}>3 L_{\text {abs }}\right)$ [28], an optimum geometry is achieved with $N_{q} \sim S_{\text {spot }}\left(P L_{\text {med }}\right)^{2}$ [10].

A careful implementation of phase matching parameters was tested in the XUV generation. The details of the HHG setup are described in previous works $[4,16]$. Generally, up to 350 millijoule, around $20 \mathrm{fs}$ driving laser passes through a 10 -centimeter-long, $10 \mathrm{~mm}$ internal diameter glass tube. 100 psi inlet pressure argon gas was ejected from a pulsed jet (Lee Co.) and synchronized with the laser pulse. The XUV was filtered out from the NIR driving laser by the utilization of a pair of beam splitters and aluminum foils. The pair of tantalum pentoxide beam splitters were placed orthogonally with 75-degree angle of incidence, and ND filters attached behind the beam splitter by thermal glue were used to absorb the transmitted NIR beam. XUV signal was detected by an XUV photodiode (AXUV100). The photodiode was calibrated, and it gives absolute XUV photon counts at the generation location considering the photodiode response efficiency and the XUV propagation throughput.

A comparison was performed between XUV counts from a small focal spot $(780 \times 780 \mu \mathrm{m})$ and a spot before the focus $(1.2 \times 1.2 \mathrm{~mm})$. The result is shown in figure 5 . It is noted that the laser peak intensity was calculated due to the beam spot size, the pulse energy and the pulse duration difference in both cases. The phase matching parameters including pulse duration, gas pressure, etc. were checked. The driving laser peak intensity matters. In both cases, the XUV signals increase with the laser intensity but saturation occurs at a lower intensity when the laser beam is optimized at the focus. The XUV threshold amount is 1.7 times higher for the off-focus spot. It is consistent with the fact that the XUV amount is proportional to the generation volume. Up to $5.6 \mu \mathrm{J}$ XUV pulse energy was obtained using a larger off-focus spot. The generated XUV

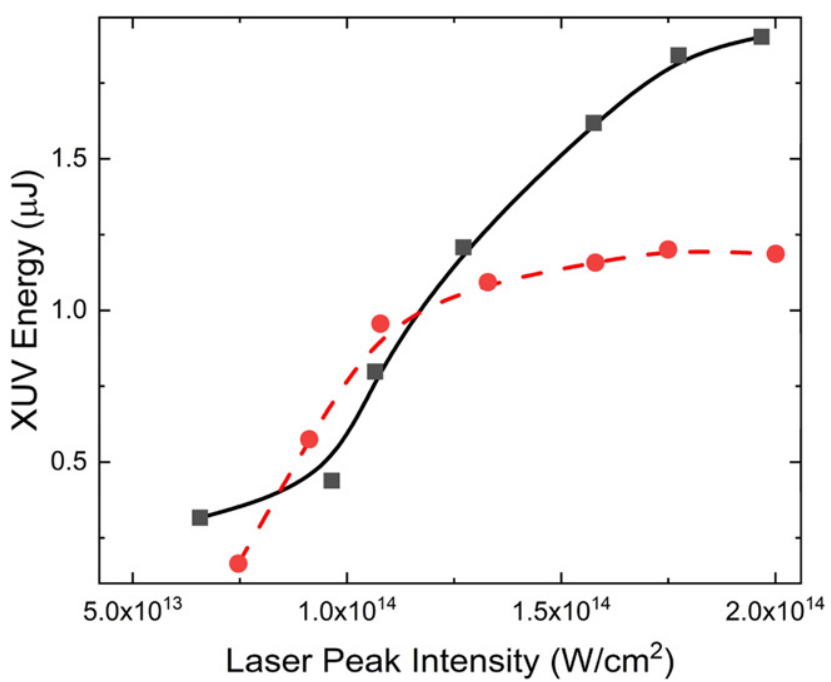

Figure 5. A comparison of XUV generation between implementation of the focal spot (red dash line, $780 \times 780 \mu \mathrm{m}$ ) and an off-focus spot (black solid line, $1.2 \times 1.2 \mathrm{~mm}$ ). With phase matching, the XUV pulsed energy is plotted versus the laser peak intensity in both cases. The data dots are connected using the B-spline.

pulse energy is improved by one order of magnitude compared to our previous work $(0.3 \mu \mathrm{J})$ [16]. The employment of even a larger off-focus spot is under investigation, where tens of $\mu \mathrm{J}$ XUV pulse energy is expected. Up to $\mu \mathrm{J}$ level isolated attosecond pulses at the sample target are promised, which will strongly support the forthcoming nonlinear absorption investigations $[29,30]$ by attosecond pulses.

\section{Conclusions}

A larger focal spot with a long focal depth is required to push the XUV pulse energy from HHG, driven by $>100 \mathrm{~mJ}$ femtosecond lasers. We introduce a way to optimize the beam spot at off-focus locations of a $25 \mathrm{TW}$ laser by adding spherical aberrations and a careful optimization. Smooth spots with $>1$ $\mathrm{mm}$ diameter were obtained both before and after the focus. We demonstrated the XUV pulse energy can be significantly increased by implementing this scheme. This method enlarges the generation volume up to one order of magnitude, which is adaptable to a joule level driving laser.

\section{Acknowledgments}

This work has been supported by the DARPA PULSE program by a grant from AMRDEC(W31P4Q-13-1-0017), Army Research Office (W911NF-14-1-0383, W911NF-19-1-0224), Air Force Office of Scientific Research (FA9550-15-1-0037, FA9550-16-1-0013, FA9550-14-1-0154), National Science Foundation (1806575), and the WM Keck Foundation No. 046300

\section{ORCID iDs}

Jialin Li (D) https://orcid.org/0000-0003-3786-004X 


\section{References}

[1] Hentschel M et al 2001 Attosecond metrology Nature 414 509

[2] Paul P M, Toma E, Breger P, Mullot G, Augé F, Balcou P, Muller H and Agostini P 2001 Observation of a train of attosecond pulses from high harmonic generation Science 292 1689-92

[3] Krausz F and Ivanov M 2009 Attosecond physics Rev. Mod. Phys. 81163

[4] Wu Y, Cunningham E, Zang H, Li J, Chini M, Wang X, Wang Y, Zhao K and Chang Z 2013 Generation of high-flux attosecond extreme ultraviolet continuum with a $10 \mathrm{TW}$ laser Appl. Phys. Lett. 102201104

[5] Li J, Ren X et al 2017 53-attosecond X-ray pulses reach the carbon K-edge Nat. Commun. 8186

[6] Lu R-F, Zhang P-Y and Han K-L 2008 Attosecond-resolution quantum dynamics calculations for atoms and molecules in strong laser fields Phys. Rev. E 77066701

[7] Santra R, Yakovlev V S, Pfeifer T and Loh Z-H 2011 Theory of attosecond transient absorption spectroscopy of strong-field-generated ions Phys. Rev. A 83033405

[8] Ferrari F, Calegari F, Lucchini M, Vozzi C, Stagira S, Sansone G and Nisoli M 2010 High-energy isolated attosecond pulses generated by above-saturation few-cycle fields Nat. Photon. 4875

[9] Sansone G, Poletto L and Nisoli M 2011 High-energy attosecond light sources Nat. Photon. 5655

[10] Boutu W, Ducousso M, Hergott J-F and Merdji H 2015 Optical Technologies for Extreme-Ultraviolet and Soft X-ray Coherent Sources (Berlin: Springer) pp 63-78

[11] Rivas D et al 2016 Generation of high-energy isolated attosecond pulses for XUV-pump/XUV-probe experiments at $100 \mathrm{eV}$ High-Brightness Sources and Light-Driven Interactions (Washington, DC: Optical Society of America)

[12] Chang Z 2016 Fundamentals of Attosecond Optics (Boca Raton, FL: CRC Press)

[13] Baumert T, Brixner T, Seyfried V, Strehle M and Gerber G 1997 Femtosecond pulse shaping by an evolutionary algorithm with feedback Appl. Phys. B 65 779-82

[14] Omenetto F G, Luce B P and Taylor A J 1999 Genetic algorithm pulse shaping for optimum femtosecond propagation in optical fibers J. Opt. Soc. Am. B 16 2005-9

[15] White J and Chang Z 2019 Attosecond streaking phase retrieval with neural network Opt. Express 27 4799_ 807
[16] Wang Y et al 2018 Enhanced high-order harmonic generation driven by a wavefront corrected high-energy laser J. Phys. B: At. Mol. Opt. Phys. 51134005

[17] Jiang W and Li H 1990 Hartmann-Shack wavefront sensing and wavefront control algorithm Proc. SPIE 1271 pp 82-93

[18] Booth M J, Neil M A, Juškaitis R and Wilson T 2002 Adaptive aberration correction in a confocal microscope Proc. Natl Acad. Sci. 99 5788-92

[19] Pittman M, Ferré S, Rousseau J-P, Notebaert L, Chambaret J-P and Chériaux G 2002 Design and characterization of a near-diffraction-limited femtosecond 100-TW $10-\mathrm{Hz}$ high-intensity laser system Appl. Phys. B 74 529-35

[20] Fourmaux S, Payeur S, Alexandrov A, Serbanescu C, Martin F, Ozaki T, Kudryashov A and Kieffer J 2008 Laser beam wavefront correction for ultra high intensities with the 200 TW laser system at the advanced laser light source $O p t$. Express 16 11987-94

[21] Kazamias S et al 2003 Global optimization of high harmonic generation Phys. Rev. Lett. 90193901

[22] Noll R J 1976 Zernike polynomials and atmospheric turbulence J. Opt. Soc. Am. 66 207-11

[23] Wang J and Silva D E 1980 Wave-front interpretation with Zernike polynomials Appl. Opt. 19 1510-8

[24] Ammosov M, Delone N, Krainov V, Perelomov A, Popov V, Terent'ev M, Yudin G L and Ivanov M Y 1986 Tunnel ionization of complex atoms and of atomic ions in an alternating electric field Sov. Phys - JETP 6426

[25] Jeong T M, Choi I W, Hafz N, Sung J H, Lee S K, Ko D-K and Lee J 2007 Wavefront correction and customization of focal spot of 100 TW Ti: sapphire laser system Jpn. J. Appl. Phys. 467724

[26] Kempe M and Rudolph W 1993 Impact of chromatic and spherical aberration on the focusing of ultrashort light pulses by lenses Opt. Lett. 18 137-9

[27] Feng X, Gilbertson S, Mashiko H, Wang H, Khan S D, Chini M, Wu Y, Zhao K and Chang Z 2009 Generation of isolated attosecond pulses with 20 to 28 femtosecond lasers Phys. Rev. Lett. 103183901

[28] Constant E, Garzella D, Breger P, Mével E, Dorrer C, Le Blanc C, Salin F and Agostini P 1999 Optimizing high harmonic generation in absorbing gases: model and experiment Phys. Rev. Lett. 821668

[29] Chang Z, Corkum P B and Leone S R 2016 Attosecond optics and technology: progress to date and future prospects [Invited] J. Opt. Soc. Am. B 33 1081-97

[30] Vampa G, Fattahi H, Vučković J and Krausz F 2017 Attosecond nanophotonics Nat. Photon. 11 210-2 